\title{
PKM2 gene regulates the behavior of pancreatic cancer cells via mitogen-activated protein kinase pathways
}

\author{
JIAKE FENG ${ }^{1 *}$, TIELIANG MA ${ }^{2 *}$, ZHIJUN GE ${ }^{3 *}$, JIE LIN $^{4}$, WEILIANG DING ${ }^{2}$, \\ HONG $\mathrm{CHEN}^{5}$, WENJIAO ZHU ${ }^{2}$, SUJUN ZHOU ${ }^{1}$ and YONGFEI TAN ${ }^{4}$ \\ ${ }^{1}$ Department of General Surgery, ${ }^{2}$ Central Laboratory, Departments of ${ }^{3}$ Critical Care Medicine, \\ ${ }^{4}$ Cardiac and Thoracic Surgery and ${ }^{5}$ Oncology, The Affiliated Yixing Hospital of Jiangsu University, \\ Yixing, Jiangsu 214200, P.R. China
}

Received December 31, 2013; Accepted November 3, 2014

DOI: $10.3892 / \mathrm{mmr} .2014 .2990$

\begin{abstract}
The aim of the current study was to investigate the effect of the PKM2 gene on the proliferation, invasion, migration and apoptosis of Panc-1 and Sw1990 pancreatic cancer cells via its interaction with the mitogen-activated protein kinases (MAPKs) signaling pathways. The expression levels of PKM2 protein in pancreatic cancer cells and the corresponding normal tissues was determined with western blot analysis. Immunohistochemical analysis of PKM2 expression was carried out in paraffin-embedded sections of pancreatic cancer tissue. Two human pancreatic cancer cell lines were cultured in vitro, and a small interfering RNA (siRNA) was designed for the PKM2 gene and transfected into the cells. Cell proliferation was measured via an MTT assay, cell migration and invasion was measured via Transwell ${ }^{\circledR}$ chambers, and the effect of PKM2 on apoptosis was detected from B-cell lymphoma 2 (Bcl-2) and $\mathrm{Bcl}-2$-associated $\mathrm{X}$ protein expression levels. Protein expression levels of the MAPK pathway proteins extracellular signal-regulated kinase 1/2 (ERK1/2), p38 and c-Jun N-terminal kinase (JNK) and their phosphorylated forms were measured via western blot analysis. The expression level of PKM2 was significantly upregulated in the pancreatic cancer tissue compared with that of the corresponding normal tissue. Downregulation of PKM2 expression reduced the proliferation, migration and invasion of pancreatic cancer cell lines, while increasing the levels of apoptosis. Additionally, the expression levels of the phosphorylated-(p-) ERK1/2 and p-p38 of the MAPK pathway in the PKM2 siRNA
\end{abstract}

Correspondence to: Professor Yongfei Tan, Department of Cardiac and Thoracic Surgery, The Affiliated Yixing Hospital of Jiangsu University, 75 Tongzhenguan Road, Yixing, Jiangsu 214200, P.R. China

E-mail: yongfeitan@sina.com

${ }^{*}$ Contributed equally

Key words: PKM2, pancreatic cancer cells, mitogen-activated protein kinase pathways, small interfering RNA groups were markedly downregulated compared with those of the controls; however, the expression levels of ERK1/2, p38, JNK, p-p38 and p-JNK had no significantly changes compared with those of the control groups. In summary, the PKM2 gene has an important role in the proliferation, invasion, migration and apoptosis of Panc-1 and Sw1990 pancreatic cancer cells, which may be associated with the expression of ERK1/2 and p38 of the MAPK signaling cascade.

\section{Introduction}

Pancreatic cancer is a highly aggressive and devastating disease, which is fatal in $95 \%$ of patients within six months of diagnosis (1). Previous studies have made a degree of progress in utilizing improved diagnostic methods and developing novel targeted therapies, however, despite this the overall survival rate has not increased in $>10$ years (2). As such, pancreatic cancer remains the fourth highest cause of cancer-related mortalities in females and males (3). Therefore, it is necessary to investigate the underlying molecular mechanisms involved in the progression of pancreatic cancer, in order to develop novel therapeutic strategies to treat it.

It has been established that the development of pancreatic cancer is associated with a number of marked metabolic changes, including an increase in glucose consumption and lactate production, even in an oxygen rich environment. Warburg determined that this phenotype occurred in response to local hypoxia (4); however, it was revealed that persistent or cyclical hypoxia caused certain selection pressures, which leads to the upregulation of glycolysis even when oxygen is present, a phenomenon that is known as aerobic glycolysis (5). In this process, pyruvate kinase limits the rate of the glycolytic pathway, catalyzing the transfer of a high-energy phosphate group from phosphoenolpyruvate to generate pyruvate and ATP (6). This metabolic pathway drives the cellular growth and survival. While the Warburg effect has been studied thoroughly, novel mechanisms are constantly being found by researchers. As the rate-limiting enzyme in glycolysis, pyruvate kinase M2 (PKM2), has only been found to be present in embryonic, proliferating, and tumor cells, and it has been determined to be critical for the metabolism and growth of tumor cells (7). 
Sun et al (7) determined that the PI3K/AKT/mechanistic target of rapamycin (mTOR) signaling pathway was a major positive regulator of aerobic glycolysis, and that the receptor tyrosine kinase/PI3K/AKT/mTOR (RTK/PI3K/AKT/mTOR) signaling pathway had an important role in the regulation of cell metabolism, growth and survival $(8,9)$. It has been determined that mTOR upregulates the expression of PKM2 via the hypoxia-inducible factor $1 \alpha(\mathrm{HIF} 1 \alpha)$-mediated activation of transcription and the c-Myc-heterogeneous nuclear ribonucleoprotein (hnRNP)-dependent regulation of PKM2 gene splicing. Therefore, PKM2 was determined to be downstream of HIF1 $\alpha$ and c-Myc-hnRNPs of mTOR signaling, and the mTOR/HIF1 $\alpha /$ Myc-hnRNPs/PKM2 signaling cascade was revealed to have an essential role in tumorigenesis (7). In summary, PKM2 has an important role in the development of tumors.

In addition, pancreatic cancer is characterized by the constitutive activation of the mitogen-activated protein kinases (MAPKs). The activation of MAPKs upregulates certain genes that are implicated in the proliferation and survival of pancreatic cancer cells (10). Consequently, the activation of the Ras/MAPK signaling pathway is another aberrantly activated pathway in tumor cells. There are three predominant distinct MAPK pathways that have been established, including the extracellular signal-regulated kinases (ERK 1/2 or p44/p42), the c-Jun N-terminal kinases (JNKs or stress activated protein kinases), and the CSBP/RK/Mpk2 (or p38) kinase (11). Wang et al (12) have previously reported that inactivation of the tumor suppressor gene Spry2 accelerated AKT-induced hepatocarcinogenesis via activation of the MAPK and PKM2 pathways (12). However, there was little data to confirm that PKM2 was associated with the MAPK signaling pathway in this study. We hypothesized that an association between the MAPK and PKM2 pathways existed in the proliferation, migration, invasion and anti-apoptosis of pancreatic cancer cells.

The aim of the present study, was to verify whether PKM2 is associated with MAPK in the induction of the progression of pancreatic cancer. By using siRNA to downregulate the expression levels of PKM2, the subsequent proliferation, invasion, migration and apoptosis capabilities of Panc-1 and Sw1990 cells may be determined. Additionally, the protein expression levels in three major MAPK pathways were investigated following PKM 2 knockdown of the two cell lines. Hence, this study aims to investigate the potential association between the expression level of PKM2 and the three predominant MAPK pathways to uncover the possible underlying mechanism of the malignant progression of pancreatic cancer cells.

\section{Materials and methods}

Pancreatic cancer cell specimens. Tumor specimens and the paired normal pancreatic ductal tissue specimens taken from a site distant from the cancerous lesion were obtained from five patients. All of the patients provided written informed consent. This study was approved by the Medical Ethics Committee of Yixing People's Hospital (Yixing, China). None of the patients received radiotherapy or chemotherapy prior to surgery.
Cell culture. Panc-1 and Sw1990 human pancreatic cancer cells (American Type Culture Collection, Manassas, VA, USA) were maintained in Dulbecco's modified Eagle's medium (DMEM ; HyClone Laboratories, Inc., Logan, UT, USA) or L15 (HyClone Laboratories, Inc.) supplemented with $10 \%$ fetal bovine serum (FBS, Hangzhou Sijiqing Biological Engineering Materials Co. Ltd., Hangzhou, China), $100 \mathrm{u} / \mathrm{ml}$ penicillin and $100 \mathrm{mg} / \mathrm{l}$ streptomycin (Beyotime Institute of Biotechnology, Haimen, China). The cells were cultured in a humidified incubator containing 5\% $\mathrm{CO}_{2}$ at $37^{\circ} \mathrm{C}$.

Immunohistochemistry. Primary pancreatic cancer tissues near the margin of the tumor and the matched normal tissues were used to assess PKM2 expression. Sections ( $5 \mu \mathrm{m})$ of the specimens were incubated with goat anti-human PKM2 antibody (Santa Cruz Biotechnology Inc, Dallas, TX, USA) overnight at $4^{\circ} \mathrm{C}$, followed by incubation with horseradish peroxidase-conjugated donkey anti-goat antibody (Santa Cruz Biotechnology Inc.) for $1 \mathrm{~h}$ at $37^{\circ} \mathrm{C}$. Immunodetection was performed using the EnVision ${ }^{\mathrm{TM}}$ Kit (Dako North America, Inc. Carpinteria, CA, USA), using diami-nobenzidine as the chromogen.

PKM2 siRNA transfection. PKM2 siRNA was purchased from Santa Cruz Biotechnology Inc. (sc-60820), and siRNA transfection was executed using Lipofectamine 2000 (Invitrogen Life Technologies, Carlsbad, CA, USA). The optimum concentration of siRNA was $150 \mathrm{~nm}$ after a period of $6 \mathrm{~h}$. Cells were collected after $48 \mathrm{~h}$, nonsense siRNA was used as the negative control and blank control.

MTT assay. Cell proliferation was measured using an MTT assay. Cells were collected $5 \mathrm{~h}$ after transfection, and plated onto 96-well plates at a density of $2 \times 10^{4}$ cells/well in DMEM and L15 containing $10 \%$ FBS. Five duplicate wells were set up for each group, including the negative control and untreated (Panc-1 and Sw1990) groups, and the test was repeated three times. After 48 h, $20 \mu$ l MTT (M2128; Sigma-Aldrich, St. Louis, MO, USA) in PBS (5 mg/ml) was added to each well, and the cells left for 4 h. An Infinite F50 Microplate Reader (TECAN, Maenndorf, Switzerland) was used to read absorbance of each well at a wavelength of $570 \mathrm{~nm}$. The optical density (OD) was used to plot proliferation curves and compare the growth of the two cell lines prior and subsequent to transfection.

Transwell $^{\circledR}$ assay. Cell migration and invasion were determined using a Transwell ${ }^{\circledast}$ (Costar, Corning Incorporated, Corning, New York, NY, USA) with a pore size of $0.8 \mu \mathrm{m} .100 \mu \mathrm{l}$ of Matrigel $^{\mathrm{TM}}$ (BD Biosciences, Franklin Lakes, NJ, USA) was placed into a 24-well Transwell ${ }^{\circledR}$ plate for the cell migration assay, and a Matrigel ${ }^{\mathrm{TM}}$-coated plate was used for the cell invasion assay. Cells $\left(2 \times 10^{5} / \mathrm{ml}\right)$ were seeded in the upper chamber, while DMEM with $10 \%$ FBS was added to the lower chamber. Following a $24-\mathrm{h}$ incubation at $37^{\circ} \mathrm{C}$, cells in the upper chamber were carefully removed with a cotton swab and the cells that had traversed to reverse face of the membrane were fixed in methanol, stained with Giemsa (Sangon, Shanghai, China), and counted.

Western blot analysis. The proteins were extracted from the two cell lines at 72-h post-transfection using RIPA lysate 

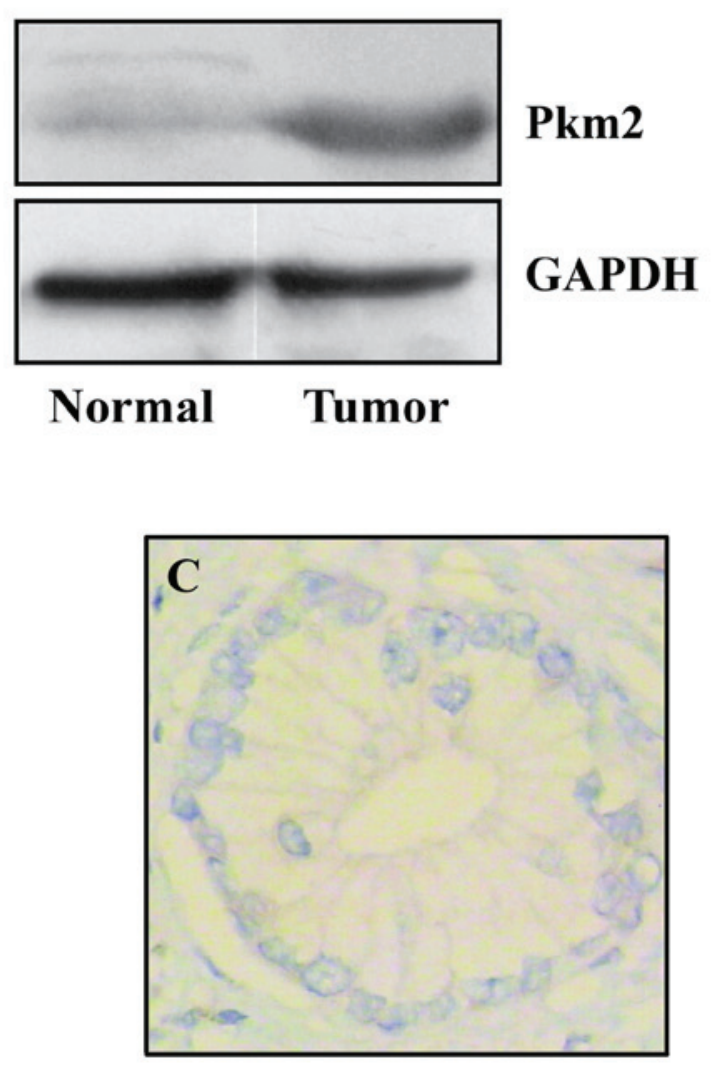

B
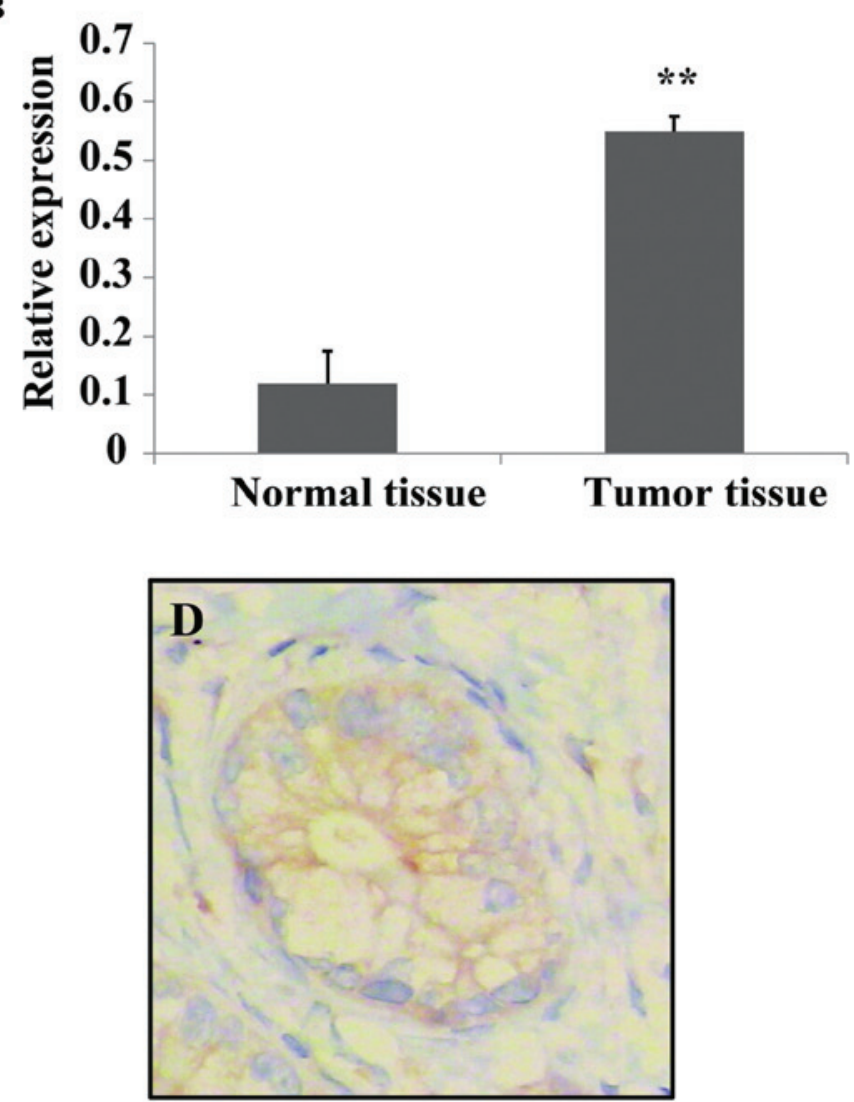

Figure 1. Comparison of the expression levels of PKM2 in pancreatic tumor and normal tissues. The expression of PKM2 in tumor and normal tissue was measured using western blotting and immunohistochemical analysis. (A and B) PKM2 expression levels were significantly higher in tumor tissues compared with those of normal tissues. (C and D) PKM2 expression was significantly higher in pancreatic cancer cells compared with normal cells $(\mathrm{P}<0.05)$.

(Beyotime), and then equal amounts $(40 \mu \mathrm{g})$ were added to each well and separated by SDS-PAGE. Following transfer to a Hybond ECL nitrocellulose membrane (GE Healthcare Life Sciences, Shanghai, China), the membrane was sealed with $5 \%$ skim milk powder at room temperature $\left(15-25^{\circ} \mathrm{C}\right)$ for $1 \mathrm{~h}$, and incubated at $4^{\circ} \mathrm{C}$ overnight $(15-17 \mathrm{~h})$ with rabbit polyclonal B-cell lymphoma2 (Bcl-2), Bcl-2-associated X protein (BAX) (Abcam), MEK, ERK1/2, p38, JNK, phosphorylated-(p-)MEK, p-ERK1/2, p-p38 and p-JNK (Cell Signaling Technology, Inc. Danvers, MA, USA) antibodies and mouse anti-human GAPDH monoclonal antibody (Beyotime) respectively.

The nitrocellulose membrane was washed after 15-17 h, incubated in the immunoglobulin $\mathrm{G}$ ( $\mathrm{IgG}$ ) secondary antibody (Merck \& Co., Inc., Whitehouse Station, NJ, USA), marked by alkaline phosphatase and stained with enhanced chemiluminescence (Lumi-Phos WB; 34150; Thermo Fisher Scientific, Tewksbury, MA, USA) at room temperature. The membrane was scanned for the relative value of protein expression. Protein levels were quantified relative to tubulin, and the software used for was Gel-Pro analyzer (version 4.0; Media Cybernetics Inc., Rockville, MD, USA).

Statistical analysis. Statistical significance was tested using SPSS version 14.0 software (SPSS, Inc. Chicago, IL, USA). Data are presented as the mean \pm standard deviation $(\bar{x} \pm \mathrm{s})$, using student $t$ tests or one-way ANOVA. $\mathrm{P}<0.05$ was considered to indicate a statistically significant difference.

\section{Results}

Pancreatic cancer tissues and cell lines expressed high levels of PKM2. Harris et al (6) reported that PKM2 was highly expressed in pancreatic cancer cells and promoted the proliferation and survival in tumor cells. In the present study, the expression of PKM2 was measured in pancreatic tumor tissue and normal tissue using western blotting analysis. It was determined that PKM2 expression levels were significantly higher in tumor tissues compared with those of normal tissues (Fig. 1A and B). In addition, the expression level of PKM2 was evaluated in tumor tissue and the matched normal pancreatic tissues via immunohistochemistry. It was determined that the expression levels of PKM2 in pancreatic cancer cells were increased compared with those of normal cells (Fig. 1C and D). These data suggest that pancreatic cancer cells have the characteristic of high expression levels of PKM2.

siRNA downregulates the expression of PKM2 in Panc-1 and Sw1990 cells. Previous experiments confirmed that PKM2 in pancreatic cancer cells was highly expressed. PKM2 siRNA was prepared to be transfected into Panc-1 and Sw1990 cells, and in addition a negative control group and a blank control group were established. The expression level of PKM2 in each group was measured with a western blotting assay. The results revealed that the expression of PKM2 in siRNA-transfected Panc-1 and Sw1990 cells were significantly lower than those 


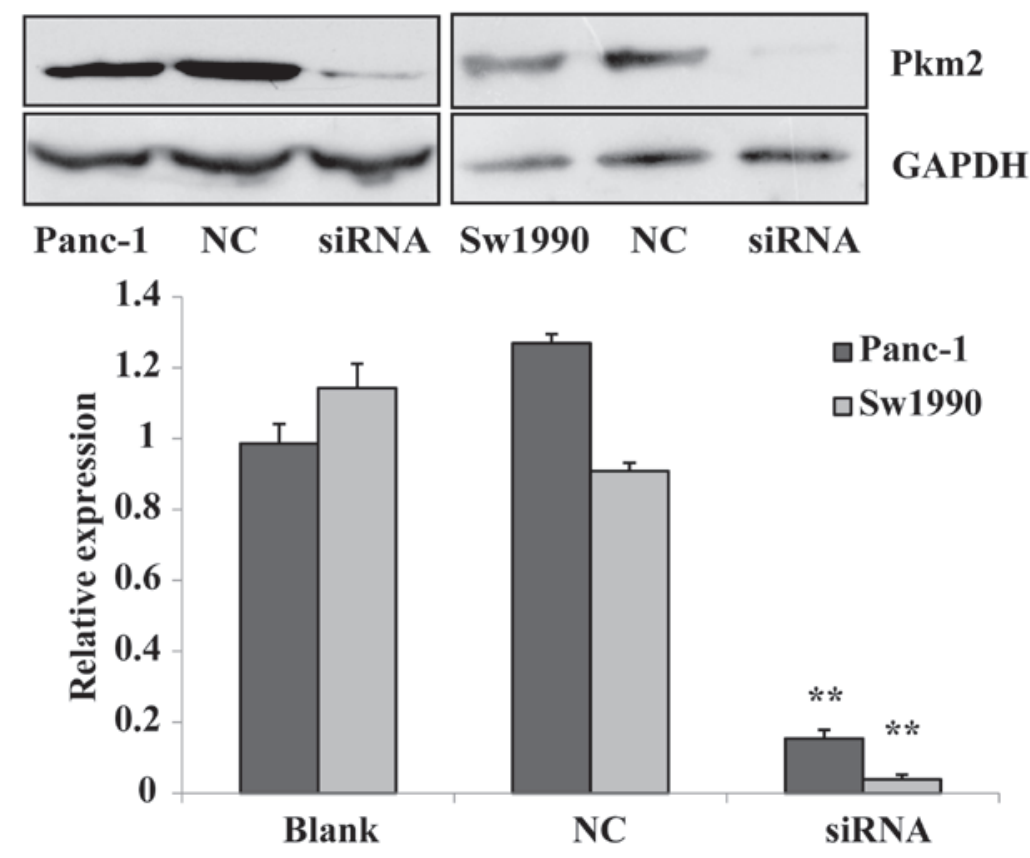

Figure 2. Effect of PKM2 siRNA transfection. A specific siRNA cell culture model was used to investigate whether PKM2 is involved in the effect of siRNA in Panc-1 and Sw1990 cells. The specific siRNA and the NC siRNA were transiently transfected into Panc-1 and Sw1990 cells. Western blot analysis showed clearly that the specific siRNA suppressed the expression of PKM2 significantly in the two cell types $(\mathrm{P}<0.01)$. NC, negative control; siRNA, small interfering RNA.
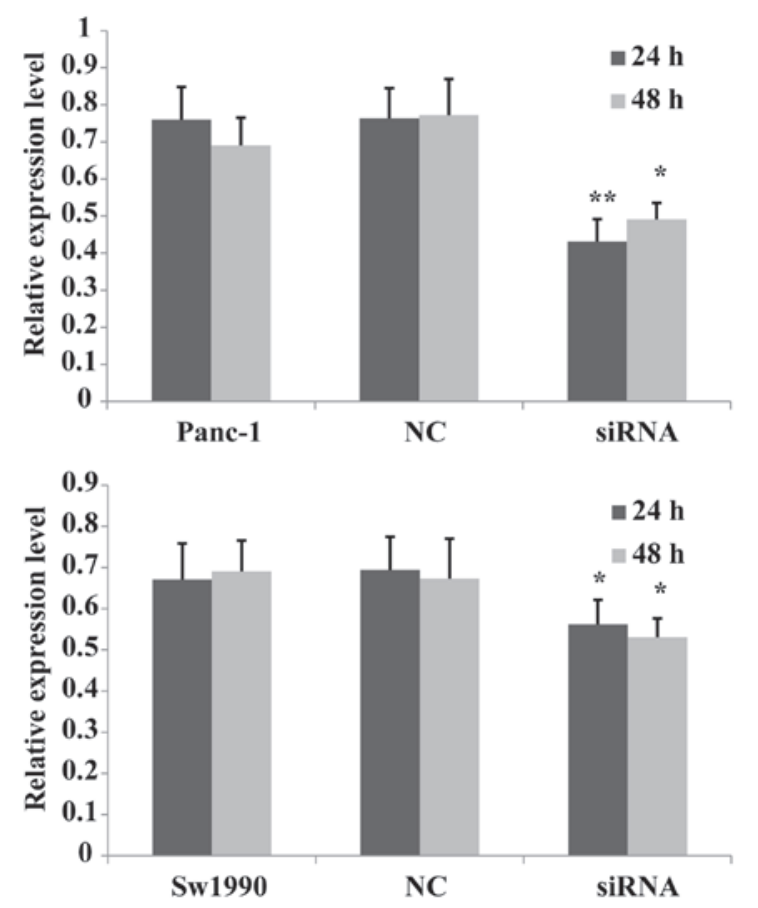

Figure 3. Proliferation of Panc-1 and Sw1990 cells following PKM2 siRNA transfection. Panc-1 and Sw1990 cells underwent an MTT assay at 24 or $48 \mathrm{~h}$ following siRNA transfection and the expression was determined by the absorbance of each well. The proliferation of the two cell lines transfected with siRNA was slightly lower than that of the other two groups, but no significant difference was identified between those treated at 24 and $48 \mathrm{~h}$ ('P>0.05). NC, negative control; siRNA, small interfering RNA.

of the negative and blank controls $(\mathrm{P}<0.01)$. This indicates that siRNA silenced the expression of PKM2, and that the inhibitory effect on PKM2 in Sw1990 cells was stronger than that in Panc-1 cells (Fig. 2). The transfection of siRNA to Panc-1 and Sw1990 pancreatic cancer cells as subject matter in our experiment.
Knocking down PKM2 decreases the proliferation of pancreatic cancer cells. A previous study discovered that the overexpression of PKM2 promoted cell proliferation in colon cancer cells, while the knockdown of PKM2 inhibited their proliferation (13). In order to determine whether PKM2 had the same effect in pancreatic cells, the current study investigated the effects of siRNA on PKM2 expression using an MTT assay in the Panc-1 and Sw1990 cell lines. The suppressive effects of PKM2 knockdown on Panc-1 and Sw1990 cell proliferation were clear after $24 \mathrm{~h}$, compared with that of the negative and blank controls. The cell proliferation of the two cell lines transfected with PKM2 siRNA was observed after $48 \mathrm{~h}$ and no significant difference was found between 24 and $48 \mathrm{~h}$ in either cell line (Fig. 3). The result indicates that PKM2 knockdown downregulated the proliferation of pancreatic cancer cells, and siRNA transfection has no significant time-dependent effect on cell proliferation.

The effect of migration and invasion following PKM2 knockdown in pancreatic cancer cells. In a previous study, it was reported that PKM2 promoted cell migration and invasion in the AGS undifferentiated gastric carcinoma cell line, which lacks E-cadherin expression (14). Another study reported that PKM2 expression is high in colorectal cancer cells, and that the knockdown of PKM2 reduced the proliferation and migration of RKO colon cancer cells (13). In the current experiment, the migration and invasion capabilities of Panc-1 and Sw1990 cells was measured using a Transwell ${ }^{\circledR}$ assay. It was revealed that the Panc-1 cell migratory and invasive abilities were markedly reduced after siRNA transfection compared with that of the negative and blank control groups. In Sw1990 cells, the migration of the siRNA group was significantly decreased compared with that of the other two groups $(\mathrm{P}<0.01)$, however, the invasion showed no significantly changes (Fig. 4). These results indicate that PKM2 transfection reduces the of migration and invasion capabilities of Panc-1 cells and the migratory ability of Sw1990 

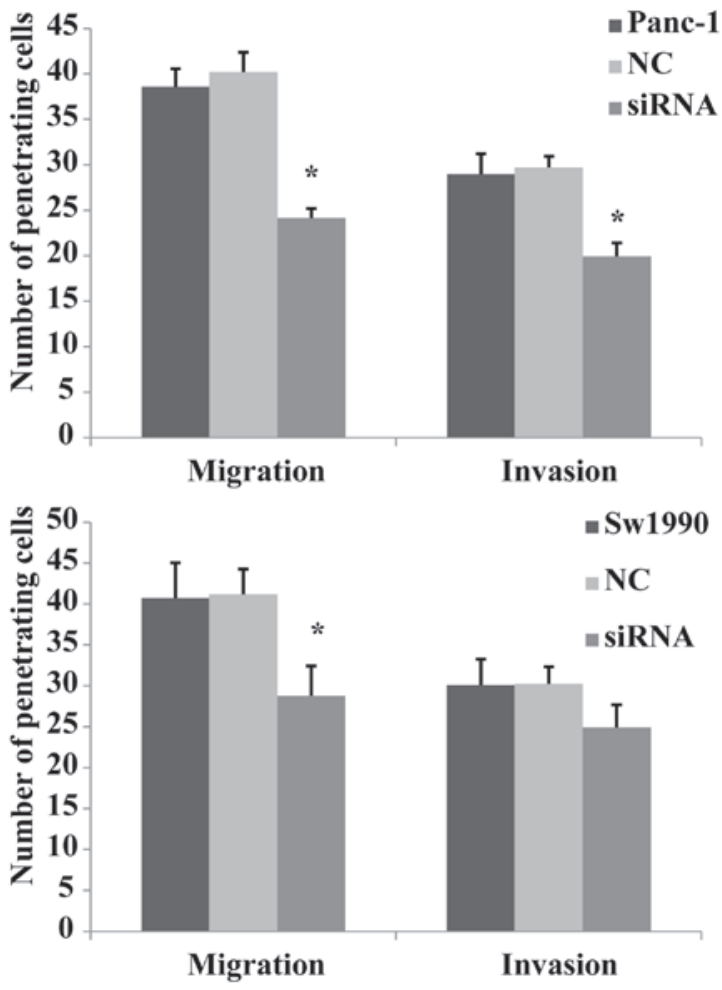

Figure 4. Effects of invasion and migration following PKM2 siRNA transfection. Transwell ${ }^{\circledR}$ chambers were used to detect cell invasion with Matrigel ${ }^{\mathrm{TM}}$ while detecting cell migration without Matrigel ${ }^{\mathrm{TM}}$. The cells migrating to the lower chambers were observed with a light microscope at x200 magnification. In Panc-1 cells, the penetrating cell number of the siRNA PKM2 group was significantly decreased in the non-basement membrane chamber and Matrigel $^{\mathrm{TM}}$-coated chamber. It was also observed that in the Sw1990 cells, the penetrating cell number of the siRNA PKM2 group in the non-basement membrane chamber was markedly decreased, however the siRNA PKM2 group in the Matrigel ${ }^{\mathrm{TM}}$-coated chamber exhibited no significant change. It was indicated that the migration and invasion of Panc-1 cells and the migration of Sw1990 decreased notably with PKM2 expression knockdown, but the invasion of Sw1990 cells exhibited no clear change. ${ }^{*} \mathrm{P}<0.01$ compared with the $\mathrm{NC}$ and blank control groups. NC, negative control; siRNA, small interfering RNA.

cells, while having no clear effect on the invasion of Sw1990 cells.

PKM2 knockown affects the levels of apoptosis in Panc-1 and Sw1990 cells. A previous study revealed that pyruvate kinase M2-specifc siRNA reduces the viability and increases apoptosis in multiple cancer cell lines, but less so in normal fibroblasts or endothelial cells (15). It has been reported that $\mathrm{BAX}$ and $\mathrm{Bcl}-2$ can independently regulate apoptosis. The $\mathrm{Bcl}-2$ protein, as one member of the Bcl-2 family, inhibits cell death, while BAX protein promotes cell apoptosis (16). In the current study, the effect of downregulating the expression of PKM2 on apoptosis in Panc-1 and Sw1990 cells was investigated. The expression levels of $\mathrm{BAX}$ and $\mathrm{Bcl}-2$ were measured using a western blot assay, and the results showed that the level of BAX protein expression in the siRNA-treated group was significantly upregulated compared with that of the negative and blank control groups, and $\mathrm{Bcl}-2$ protein expression in siRNA group was significantly downregulated compared with that of the other two groups (Fig. 5). The results showed that downregulating the expression level of PKM2 promotes the downregulation of the expression of BAX protein and the upregulation of the expression of $\mathrm{Bcl}-2$ protein.
Association between PKM2 expression level and the MAPK signaling pathway. Through immunohistochemical analyses, Wang et al (14) have shown that the levels of E-cadherin expression, ERK1/2 phosphorylation and cytoplasmic PKM2 expression are correlated with each other. Furthermore, they demonstrated a high level of ERK1/2 phosphorylation without E-cadherin expression but with a high level of PKM2 expression in the nucleus of gastric cancer cells (14). Based on the results of previous studies, the current study investigated whether the expression level of PKM2 would have an effect on the MAPK signaling pathway in pancreatic cancer, using western blotting to measure the phosphorylated and non-phosphorylated proteins of the MAPK in Panc-1 and Sw1990 cells treated with siRNA. The results revealed that when the expression level of PKM2 in Panc-1 and Sw1990 cells was reduced, p-ERK1/2 and p-p38 expression levels were significantly reduced in the MAPK signaling pathway, while the ERK1/2, p38, JNK, p-JNK expression were not significantly changed. The results indicated that knockdown of PKM2 inhibited the MEK-ERK and p38 signaling pathways, however, it had no significant effect on the JNK pathway (Fig. 6). Therefore, we suspected that PKM2 regulated certain biological functions associated with of progression of pancreatic cancer cells through MAPK pathway.

\section{Discussion}

Pancreatic cancer is a common malignancy around the world, the development of which is a multi-step process whereby multiple pathways are deregulated. One of the primary hallmarks of pancreatic cancer is its early systemic dissemination and its accelerated local tumor progression. However, these characteristics closely correlate with poor clinical prognosis and represent a formidable barrier to successful treatment.

In a previous study, researchers discovered that the $\mathrm{PI} 3 \mathrm{~K} / \mathrm{AKT} / \mathrm{mTOR}$ signaling pathway is a major positive regulator of the Warburg effect, a hallmark of cancer metabolism. This signaling pathway has an important role in the regulation of the progression of tumors (17). PKM2 is a major effector of mTOR signaling, downstream of HIF1 $\alpha$ and the c-Myc-hnRNPs. The mTOR/HIF1 $\alpha /$ Myc-hnRNPs/PKM2 signaling cascades are critical for oncogenic mTOR-mediated tumorigenesis (7). In the current study, siRNA was utilized to knockdown PKM2 in human pancreatic cancer cells, in order to observe their proliferation, apoptosis, migration and invasion capabilities. Subsequently, it was investigated whether the expression level of PKM2 was associated with the activation of the MAPK signal pathway.

A previous study suggested that overexpression of PKM2 promotes the proliferation and migration of colon cancer cells (14). At the same time, researchers have demonstrated that the knockdown of PKM2 using specific siRNA inhibited the proliferation and invasion of cancer cell in vitro and the formation of xenograft tumors in vivo $(18,19)$. In the present study, it was determined that in Panc-1 and Sw1990 cells, PKM2 knock-down downregulates the proliferation of pancreatic cancer cells compared to that of the normal pancreatic tissue, however, there was no significant time-dependent effect on cell proliferation in either cell line. Subsequently, the migration and invasion capabilities of the siRNA groups was 
A

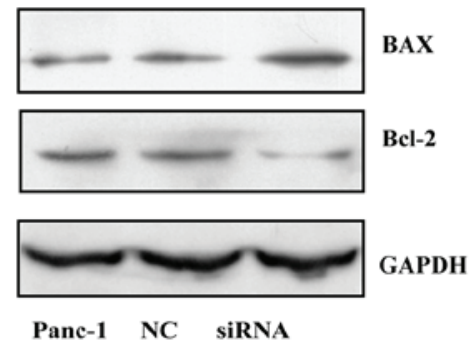

B

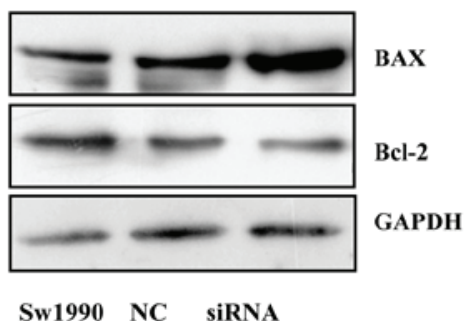

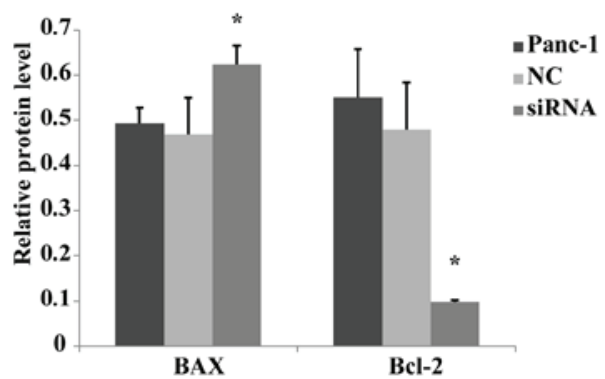

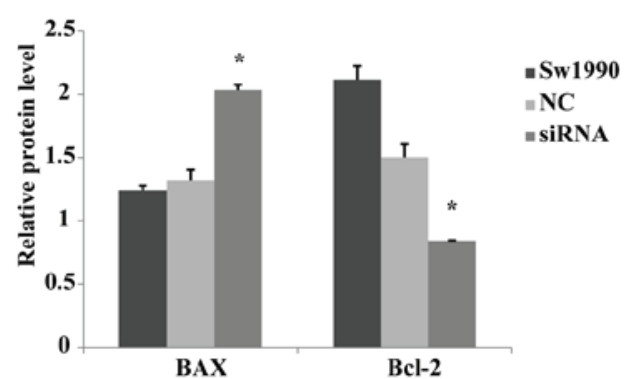

Figure 5. Effects of PKM2 on siRNA-induced cell apoptosis. Western blot analysis was used to examine the BAX and Bcl-2 protein expression of the siRNA, NC and blank control groups to determine the apoptosis effect of PKM2 siRNA transfection in (A) Panc-1 and (B) Sw1990 cells. It was revealed that the expression level of BAX protein was significantly upregulated and the expression level of $\mathrm{Bcl}-2$ protein was downregulated in the two cell types $(\mathrm{P}<0.05)$. $\mathrm{NC}$, negative control; siRNA, small interfering RNA; Bcl-2, B-cell lymphoma 2; BAX, Bcl-2-associated X protein.

$\mathbf{A}$
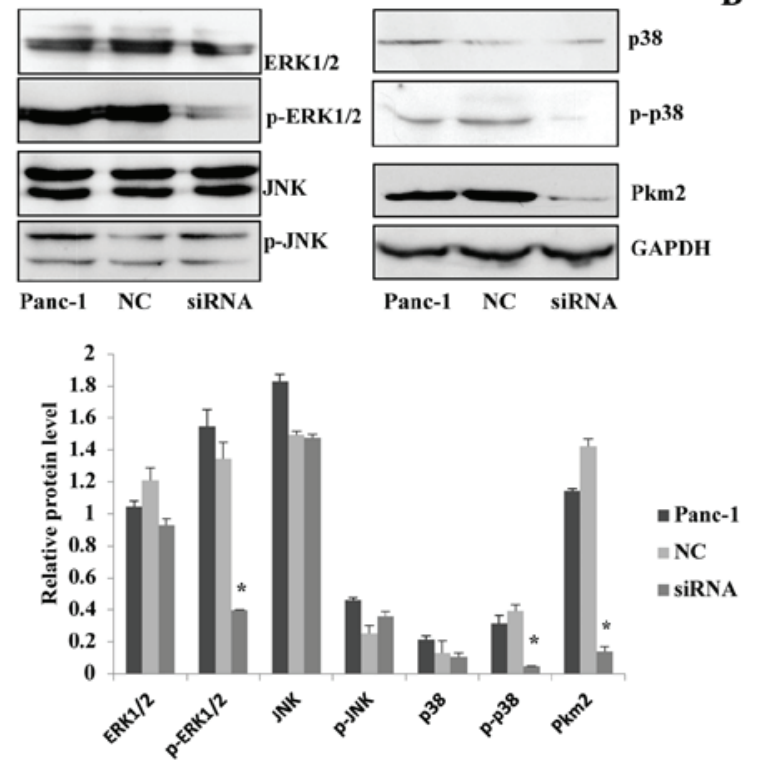

B
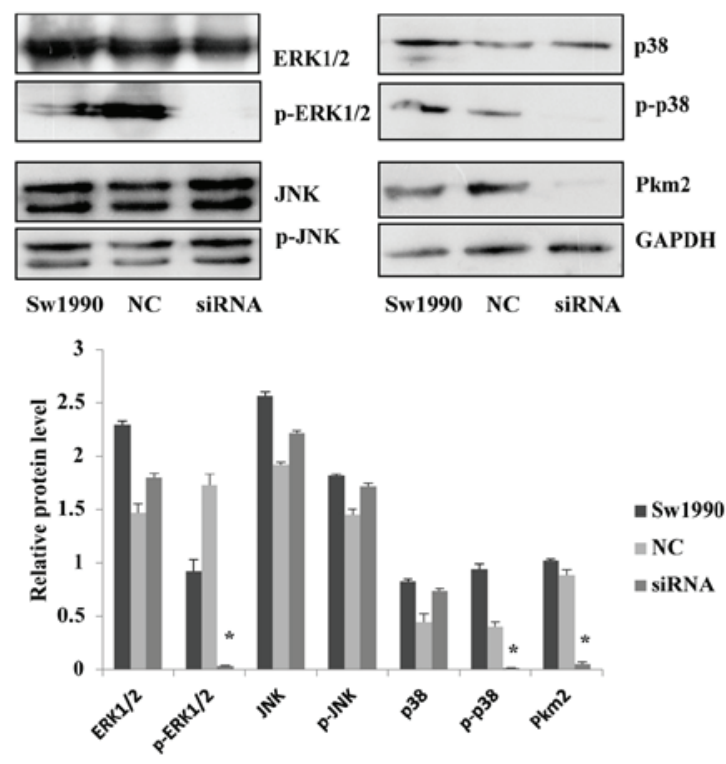

Figure 6. Protein expression level of the MAPK pathway following transfection with PKM2 siRNA in (A) Panc-1 and (B) Sw1990 cells. It was shown that the expression levels of p-ERK1/2 and p-p38 were markedly downregulated and that the levels of ERK1/2 and p38 showed little change. In another pathway of the MAPK signaling cascade, the expression of p-JNK and JNK showed no significant change $(\mathrm{P}<0.05)$. MAPK, mitogen-activated protein kinase; NC, negative control; siRNA, small interfering RNA; p-, phosphorylated-; ERK, extracellular signal-regulated kinase; JNK, c-Jun N-terminal kinase.

determined in the two cell lines. In Panc-1 cells, the migration and invasion abilities were significantly reduced after siRNA transfection. Notably, in Sw1990 cells, the migration of the experimental group was significantly reduced, however, the invasion showed no significantly changes. This phenomenon is worth researching and discussing in the future.

Kwon et al have previously reported that the apoptotic pathway is involved in the reduced cell growth after PKM2 knockdown in gastric cancer cells (20). The current study utilized siRNA transfection to downregulate the expression of PKM2 in pancreatic cancer cells. It was revealed that the expression level of apoptosis-associated protein BAX increased markedly, and the expression level of Bcl-2 was reduced significantly. Therefore, it is indicated that the low expression of PKM2 may promote the apoptosis of tumor cells, but has no significant effect on normal pancreatic tissue.

Wang et al have documented that activation of the MAPK and PKM2 pathways may accelerate hepatocarcinogenesis, 
and PKM2 activation is independent of MAPK or AKT/mTOR cascades (12). Another study demonstrated that a high level of ERK1/2 phosphorylation was associated with a high level of PKM2 expression in gastric cancer cells (14). In order to determine the association between PKM2 and MAPK in the present study, the protein expression levels were detected in three major pathways of the MAPK signaling pathway after PKM2 knockdown in pancreatic cancer. In addition, it was determined that the expression level of $\mathrm{p}-\mathrm{ERK} 1 / 2$ was reduced significantly in the experimental group, but the levels of non-phosphorylated ERK1/2 had no significant change. Similarly, significant changes appeared in the P38 signaling pathway. It was revealed that the level of p-p38 protein was reduced in the siRNA group following PKM2 knockdown, but the levels of non-phosphorylated p38 had no significant change. However, the expression levels of non-phosphorylated JNK and p-JNK did not change in Panc-1 and Sw1990 cells after siRNA PKM2 infection. PKM2 knockdown did reduce the levels of phosphorylation of ERK1/2 and p38. Therefore, we hypothesized that PKM2 has an important role in regulating certsin proteins in the MEK-ERK1/2 and p38 signaling pathways in the two cell lines.

In conclusion, the glycolytic rate-limiting enzyme PKM2 has an essential role in the proliferation, apoptosis, migration and invasion of Panc-1 and Sw1990 pancreatic cancer cells. The downregulation of PKM2 expression inhibits the proliferation, migration and invasion and promotes the apoptosis of pancreatic cancer cells. This phenomenon may be realized via the MEK-ERK1/2 and p38 pathways in the MAPK signaling pathways. These findings provide novel scientific evidence for the tumorigenesis of pancreatic cancer.

\section{Acknowledgements}

This study was supported in part by grants from the Natural Science Foundation of Jiangsu Province (BK2012563), and the Medical Research Project of Health Department of Jiangsu Province (Z201218).

\section{References}

1. Botta GP, Reginato MJ, Reichert M, Rustgi AK and Lelkes PI: Constitutive K-RasG12D activation of ERK 2 specifically regulates 3D invasion of human pancreatic cancer cells via MMP-1. Mol Cancer Res 10: 183-196, 2012.
2. Long J, Zhang Y, Yu X, et al: Overcoming drug resistance in pancreatic cancer. Expert Opin Ther Targets 15: 817-828, 2011.

3. Siegel R, Naishadham D and Jemal A: Cancer statistics, 2013. CA Cancer J Clin 63: 11-30, 2013.

4. Tamada M, Suematsu M and Saya H: Pyruvate kinase M2: multiple faces for conferring benefits on cancer cells. Clin Cancer Res 18: 5554-5561, 2012.

5. Kumar Y, Mazurek S, Yang S, et al: In vivo factors influencing tumour M2-pyruvate kinase level in human pancreatic cancer cell lines. Tumour Biol 31: 69-77, 2010.

6. Harris I, McCracken S and Mak TW: PKM2: a gatekeeper between growth and survival. Cell Res 22: 447-449, 2012.

7. Sun Q, Chen X, Ma J, et al: Mammalian target of rapamycin up-regulation of pyruvate kinase isoenzyme type M2 is critical for aerobic glycolysis and tumor growth. Proc Natl Acad Sci USA 108: 4129-4134, 2011.

8. Moritz A, Li Y, Guo A, et al: Akt-RSK-S6 kinase signaling networks activated by oncogenic receptor tyrosine kinases. Sci Signal 3: ra64, 2010.

9. Govindarajan B, Sligh JE, Vincent BJ, et al: Overexpression of Akt converts radial growth melanoma to vertical growth melanoma. J Clin Invest 117: 719-729, 2007.

10. Furukawa T, Tanji E, Kuboki Y, et al: Targeting of MAPK-associated molecules identifies SON as a prime target to attenuate the proliferation and tumorigenicity of pancreatic cancer cells. Mol Cancer 11: 88, 2012.

11. Peti W and Page R: Molecular basis of MAP kinase regulation. Protein Sci 22: 1698-1710, 2013.

12. Wang C, Delogu S, Ho C, et al: Inactivation of Spry2 accelerates AKT-driven hepatocarcinogenesis via activation of MAPK and PKM2 pathways. J Hepatol 57: 577-583, 2012.

13. Zhou CF, Li XB, Sun H, et al: Pyruvate kinase type M2 is upregulated in colorectal cancer and promotes proliferation and migration of colon cancer cells. IUBMB Life 64: 775-782, 2012.

14. Wang LY, Liu YP, Chen LG, et al: Pyruvate kinase M2 plays a dual role on regulation of the EGF/EGFR signaling via E-cadherin-dependent manner in gastric cancer cells. PLoS One 8: e67542, 2013.

15. Goldberg MS and Sharp PA: Pyruvate kinase M2-specific siRNA induces apoptosis and tumor regression. J Exp Med 209: 217-224, 2012.

16. Reed JC: Proapoptotic multidomain Bcl-2/Bax-family proteins: mechanisms, physiological roles, and therapeutic opportunities. Cell Death Differ 13: 1378-1386, 2006.

17. Ma J, Meng Y, Kwiatkowski DJ, et al: Mammalian target of rapamycin regulates murine and human cell differentiation through STAT3/p63/Jagged/Notch cascade. J Clin Invest 120: 103-114, 2009.

18. Kefas B, Comeau L, Erdle N, et al: Pyruvate kinase M2 is a target of the tumor-suppressive microRNA-326 and regulates the survival of glioma cells. Neuro Oncol 12: 1102-1112, 2010.

19. Goldberg MS and Sharp PA: Pyruvate kinase M2-specific siRNA induces apoptosis and tumor regression. J Exp Med 209: 217-224, 2012.

20. Kwon OH, Kang TW, Kim JH, et al: Pyruvate kinase M2 promotes the growth of gastric cancer cells via regulation of Bcl-xL expression at transcriptional level. Biochem Biophys Res Commun 423: 38-44, 2012. 